\title{
Evaluable for Safety and Efficacy
}

National Cancer Institute

\section{Source}

National Cancer Institute. Evaluable for Safety and Efficacy. NCI Thesaurus. Code C142546.

Patients who meet a clinical trial's inclusion criteria for safety analysis and efficacy evaluation. 